\title{
DRAFT
}

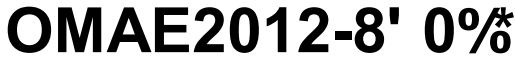

\section{EXPERIMENTAL INVESTIGATION OF THE VORTEX-INDUCED VIBRATION OF A CURVED CYLINDER}

\author{
Gustavo R.S. Assi* \\ NDF Research Group \\ Dept. Naval Arch. \& Ocean Eng. \\ University of São Paulo \\ São Paulo - SP, Brazil \\ Cesar M. Freire \\ NDF Research Group \\ Dept. Mechanical Eng. \\ University of São Paulo \\ São Paulo - SP, Brazil
}

\author{
Narakorn Srinil ${ }^{\dagger}$ \\ Dept. Naval Arch. \& Marine Eng. \\ University of Strathclyde \\ Glasgow, UK
}

\section{ABSTRACT}

Experiments have been conducted in a water channel in order to investigate the vortex-induced vibration (VIV) response of a rigid section of a curved circular cylinder. Two curved configurations were tested regarding the direction of the approaching flow, a concave or a convex cylinder, in addition to a straight cylinder that served as reference. Amplitude and frequency response are presented versus reduced velocity for a wide Reynolds number range between 750 and 15,000. Trajectories in the cross-flow and streamwise direction are presented as well for several reduced velocities. Results show a distinct behaviour from the typical VIV of a straight cylinder highlighting the effect of curvature on vortex formation and excitation. The concave configuration presents relatively high amplitudes of vibration that are sustained beyond the typical synchronisation region. The mechanism behind the response is not yet clear, although authors suggest it might be related to some kind of buffeting excitation due to the disturbed flow from

*E-mail: g.assi@usp.br. Address: PNV Dept. Eng. Naval e Oceânica, Escola Politécnica da Universidade de São Paulo, Av. Prof Mello Moraes 2231, 05508-030, São Paulo - SP, Brazil.

${ }^{\dagger}$ E-mail: narakorn.srinil@strath.ac.uk. Address: Dept. Naval Arch. \& Marine Eng., Henry Dyer Building, 100 Montrose Street, Glasgow, G4 OLZ, UK the upstream horizontal part.

Keywords: VIV - vortex-induced vibration, curved pipe.

\section{NOMENCLATURE}

$D$ Cylinder external diameter

$m^{*} \quad$ Mass ratio

$\zeta$ Structural damping ratio

$f_{0} \quad$ Natural frequency in air

$U$ Flow speed

$U / D f_{0} \quad$ Reduced velocity

$\hat{x} \quad$ Streamwise harmonic amplitude of vibration

$\hat{y}$ Cross-flow harmonic amplitude of vibration

$f_{x} \quad$ Streamwise oscillating frequency

$f_{y} \quad$ Cross-flow oscillating frequency

Re Reynolds number

St Strouhal number

\section{INTRODUCTION}

Ongoing deep-sea explorations, installations and productions of hydrocarbon energy need the development 
of new viable technologies. One of these is the requirement of a robust and completely-reliable analysis tool for the prediction of vortex-induced vibration (VIV) of marine structures exposed to ocean currents. Because VIV can cause high cyclic-loading fatigue damage of structures, it is now widely accepted that VIV is a crucial factor that should be taken into account in the preliminary analysis and design. However, many insightful VIV aspects are still unknown and far from fully understood; these render the structural design quite conservative with the use of a large factor of safety. For offshore structures with initial curvatures and high flexibility such as catenary risers, mooring cables and free-spanning pipelines, the theoretical, numerical or experimental VIV research is still very lacking.

Risers are very long pipes used to carry oil from the sea bed to offshore platforms floating on the water surface. Under the effect of sea currents, these flexible structures are especially susceptible to flow-induced vibrations, particularly since they have a relatively low mass compared to the mass of the displaced fluid. Generally, an offshore floating platform accommodates more than 40 riser pipes together with many other cylindrical structures. The interaction of these flexible structures can produce an even more complex problem, resulting in vibrations with rather unexpectedly higher amplitudes. Flow interference from the platform hull, the soil on sea bed and the pipe itself can also increase the complexity of the flow, generating complex responses.

The riser may respond with different amplitudes and frequencies depending on the flow excitation and structural stiffness along the length of the pipe. Consequently, several modes of vibration with varying curvature appear along the span resulting in a very rich fluid-structure interaction mechanism. In addition to that, flexible risers can be laid out in a catenary configuration which results in high curvature close to the region where it touches the bottom of the ocean, called the touchdown point.

In an attempt to understand and model the fluid-dynamic behaviour around curved sections of risers we have performed experiments with a curved section of rigid cylinder in a water channel. This idealised experiment is far from reproducing the real conditions encountered in the ocean, nevertheless it should throw some light on understanding how the vortex shedding mechanism and the dynamic response of the structure are affected by the curvature of the pipe.

An investigation into the vortex shedding patterns and the fundamental wake topology of the flow past a stationary curved circular cylinder has been carried out by Miliou et al. [1] based on the computational fluid dynamics studies. As a result of pipe initial curvatures, the flow visualizations highlight different kinds of wake characteristics depending on the pipe (convex or concave) configuration and its orientation with respect to (aligned with or normal to) the incoming flow. When the flow is uniform and normal to the curvature plane, the cross-flow wake dynamics of curved pipes behave qualitatively similar to those of straight pipes. This is in contrast to the case of flow being aligned with the curvature plane where wake dynamics change dramatically. However, these scenarios are pertinent to a particular stationary cylinder case in a very low-Reynolds number range. The VIV behaviors will further transform if the structure oscillates and interacts with the fluid wakes, depending on several fluid-structure parameters.

\section{EXPERIMENTAL ARRANGEMENT}

Experiments have been carried out in the Circulating Water Channel of the NDF (Fluids and Dynamics Research Group) at the University of São Paulo, Brazil. The NDF-USP water channel has an open test section $0.7 \mathrm{~m}$ wide, $0.9 \mathrm{~m}$ deep and $7.5 \mathrm{~m}$ long. Good quality flow can be achieved up to $1.0 \mathrm{~m} / \mathrm{s}$ with turbulence intensity less than $3 \%$. This laboratory has been especially designed for experiments in flow-induced vibrations and more details about the facilities are described in Assi et al. [2].

A rigid section of a curved circular cylinder, with an external diameter of $D=32 \mathrm{~mm}$, was made of ABS plastic and Perspex tubes according to the dimensions shown in Figure 1. The curved cylinder was composed of a horizontal section with $10 \mathrm{D}$ in length, a curved section with a $10 D$ radius and a vertical section with length $10 D$ measured up to the water line. The water level was set to $700 \mathrm{~mm}$ from the floor of the channel, which means that the $10 D$-long horizontal part of the cylinder was not close enough to the floor in order to suffer interference from the wall.

The model was connected by its upper end to a long pendulum rig (length $H=3.0 \mathrm{~m}$ ) that allowed the system to oscillate in two degrees of freedom (2dof) in the cross-flow and streamwise directions. The model was attached to two pairs of coil springs that provided the stiffness of the system. The springs were set to provide the same natural frequency $\left(f_{0}\right)$ in both the cross-flow and streamwise directions. The design and construction of the pendular elastic rig was made by Freire et al. [3] based on a previous idea employed by Assi et al. [4,5] for experiments with VIV suppressors. The present apparatus has been validated for VIV experiments by Freire at al. [6,7].

Two laser sensors measured the cross-flow and streamwise displacements of the pendulum on a section slightly above the water level. An especially built load cell was installed between the cylinder and the pendulum arm to allow for instantaneous measurements of lift, drag and torque acting on the cylinder. A PIV system was employed to analyse the wake along the span. (Hydrodynamic forces and PIV measurements will not be discussed in this paper.)

Regarding the flow direction, two orientations were investigated: a convex and a concave configuration according to the direction of the flow approaching the curvature. This is also illustrated in Figure 1. 


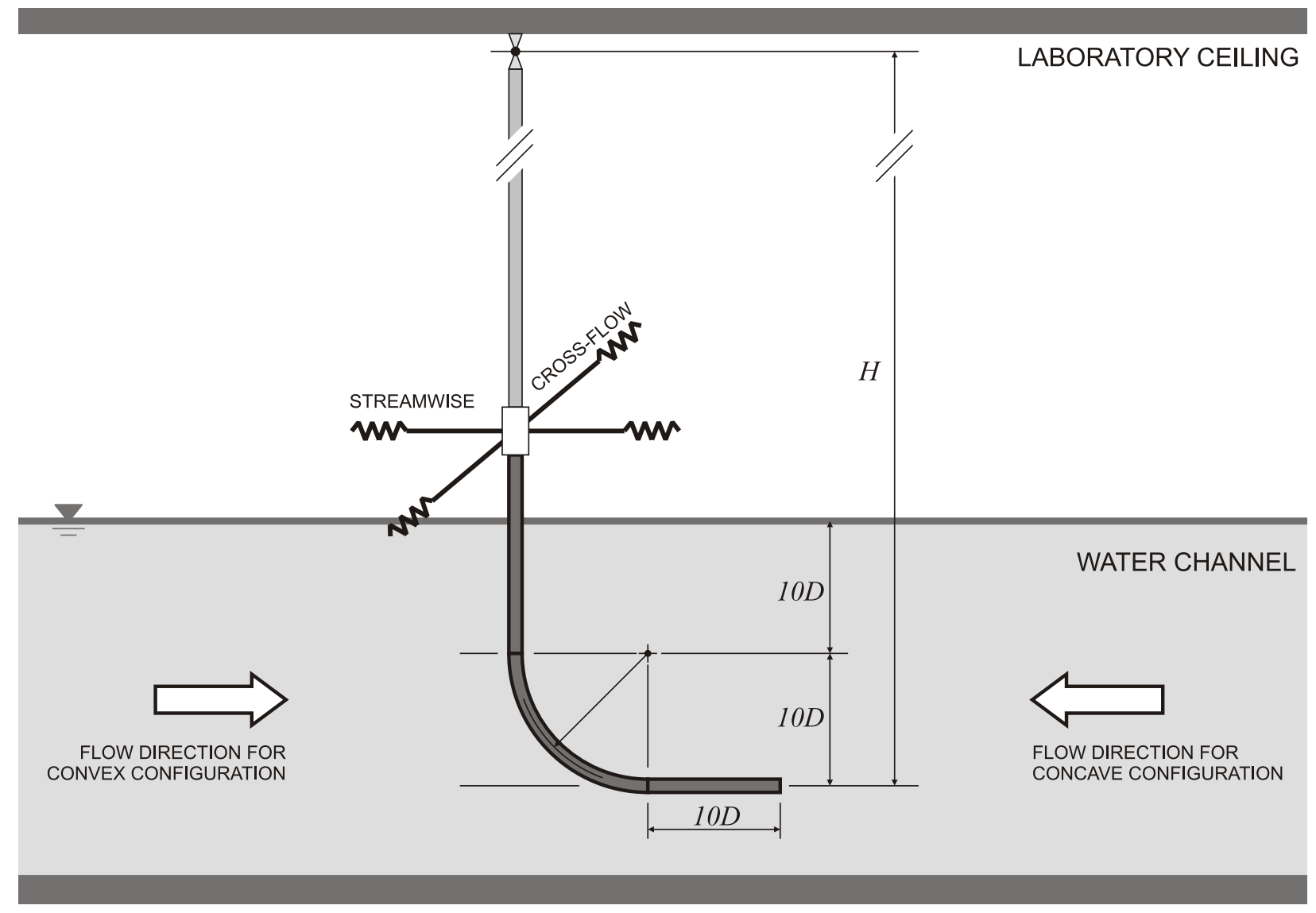

FIGURE 1. Experimental arrangement in the NDF-USP circulating water channel.

Decay tests have been performed in air in order to determine the natural frequencies of the system in both directions as well as the level of structural damping. The apparatus with one universal joint and four springs turned out to present a very low structural damping of $\zeta=0.2 \%$, measured as a fraction of the critical damping. The total oscillating mass of the system was measured in air, resulting in a non-dimensional mass parameter $m^{*}$, defined as the ratio between the total mass and the mass of displaced fluid. Consequently, the mass-damping parameter $m^{*} \zeta$ of the system was kept to the lowest possible value in order to amplify the amplitude of response.

Table 1 presents a summary of the structural parameter for both the straight and curved cylinder.

\section{PRELIMINARY RESULTS FOR A STRAIGHT CYLINDER}

A preliminary VIV experiment was performed with a straight cylinder in order to validate the set-up and methodology. The same pendulum rig was employed, only replacing the curved model by a straight cylinder with the same diameter. This time,
TABLE 1. Structural properties.

\begin{tabular}{l|ccc} 
& $m^{*}$ & $\zeta$ & $m^{*} \zeta$ \\
\hline Straight cylinder & 2.8 & $0.2 \%$ & 0.0056 \\
Curved cylinder & 2.1 & $0.2 \%$ & 0.0042 \\
\hline
\end{tabular}

the straight cylinder was long enough to reach the bottom wall only leaving a $3 \mathrm{~mm}$ clearance to allow for free movement of the pendulum in any direction.

The dynamic response of the straight cylinder covered a reduced velocity range from 1.5 to 12 , where reduced velocity $\left(U / D f_{0}\right)$ is defined using the cylinder natural frequency of oscillation measured in air. The only flow variable changed during the course of the experiments was the flow velocity $U$, which, as for full-scale risers, alters both the reduced velocity and the Reynolds number between 750 and 15,000 for a maximum reduced velocity of 20 .

Throughout the study, cylinder displacement amplitudes 
( $\hat{x} / D$ for the streamwise and $\hat{y} / D$ for the cross-flow directions) were found by measuring the root mean square value of response and multiplying by the square root of 2 (the so called harmonic amplitude). This is likely to give an underestimation of maximum response but was judged to be perfectly acceptable for assessing the general behaviour of VIV. Results presented below correspond to the displacement of the lowest point of the model, i.e, the end of the cylinder closer to the section floor, thus representing the maximum displacement developed by each model. Displacements are nondimensionalised by the cylinder diameter $D$.

Figure 2 compares the reference cross-flow and streamwise responses obtained from two different runs with the straight cylinder. In the first one, flow speed was increased in 30 steps from zero to a maximum, while in the second it was decreased from the maximum to zero. Both data sets overlap rather well for all the reduced velocity range except for a region around $U / D f_{0}=6$ where the well-known phenomenon of hysteresis in the VIV response has been observed. Although the observed peak amplitude of $\hat{y} / D=1.5$ around $U / D f_{0}=6$ is slightly higher than other results found in the literature for similar values of $m^{*}$ (for example, Assi et al. [4]) the general behaviour of both curves show a typical response for 2-dof VIV. The higher amplitude found here could be explained by the very low mass-damping characteristics of the system and the geometric projection of the amplitude at the tip of the model and not at mid-length as usual.

Although the cylinder was initially aligned in the vertical position, in flowing water the mean drag displaces the cylinder from its original location reaching a slightly inclined configuration from the vertical. This was judged not to be detrimental to the experiment, hence the inclination of the cylinder was not corrected between each step. The same procedure was adopted for the curved cylinder.

\section{RESULTS FOR THE CURVED CYLINDER}

As mentioned above, experiments with the curved cylinder were performed taking into account two distinct configurations as far as the flow direction is concerned. In the concave configuration the flow approaches the model reaching first the horizontal section. As opposed to that, in the convex configuration the horizontal section is placed downstream of the curved and vertical parts.

In general terms, as presented in Figure 2, the curved cylinder presented significant less vibration for both concave and convex configurations when compared to the typical VIV response of a straight cylinder. Such a reduction is noticeable in both the cross-flow and streamwise responses. This clearly shows that the curvature of the cylinder modifies the vortex shedding mechanism in a manner that the structure extracts less energy from the flow.
The cross-flow displacement does not reveal distinct upper and lower branches of vibration such as those observed for a straight cylinder, but it produces a smooth curve that spans the whole synchronisation region with a maximum amplitude around $\hat{y} / D=0.75$ for the concave and 0.65 for the convex configurations.

However, the most interesting feature of such a behaviour is found when the convex response is compared with the concave one. While the convex curve for $\hat{y} / D$ drops immediately between $U / D f_{0}=8$ and 10 to a level of $\hat{y} / D \approx 0.1$, the response for the concave case does not diminish, but is sustained for higher reduced velocities around $\hat{y} / D=0.3$ until the end of the experiment.

In the streamwise direction the response of the curved cylinder is distinctively different from the typical VIV developed by the straight cylinder. At the same time, the streamwise vibration $\hat{x} / D$ for the concave case also shows increasing amplitude taking off for reduced velocities higher than 10 and reaching $\hat{x} / D \approx 0.35$ for the highest flow speed.

Figure 3 presents the dominant frequency of oscillation nondimensionalised by the natural frequency for both cross-flow and streamwise directions of motion. Two dashed lines inclined with different slopes represent the region for a Strouhal number of 0.2 and 0.4 , i.e., an estimation of the vortex shedding frequency for a straight cylinder in the cross-flow and streamwise direction respectively.

Apart from a few data points at very low reduced velocities (small amplitudes result in broader spectrum analysis), the cross-flow frequency of oscillation for the straight cylinder follows the expected behaviour along the Strouhal line, departing from it towards unity for reduced velocities beyond the resonance peak. The streamwise dominant frequency is also in agreement with other results in the literature and follows the line with double the Strouhal frequency for the initial and upper branches of cross-flow vibration.

Results for the curved cylinder show a consistent behaviour in the cross-flow direction, with data points following the Strouhal line up to the upper branch peak but remaining closer to $f_{y} / f_{0}=1.0$ for the rest of the reduced velocity range. In the streamwise direction, we find data points following both Strouhal lines and also very low frequencies indicating random drifts instead of periodic oscillations. Since the displacements in the streamwise direction are much smaller for the curved cylinder than the straight one, we should expect broader frequency spectra dominating over the response.

One might remember that the straight and curved cylinder should have very similar values of added mass in the cross-flow direction, but slightly different values in the streamwise direction due to the geometric properties. We have not taken such effect into account in this paper, but it might be playing an important role defining the frequencies of oscillation in water.

Figure 4 qualitatively compares samples of displacement 

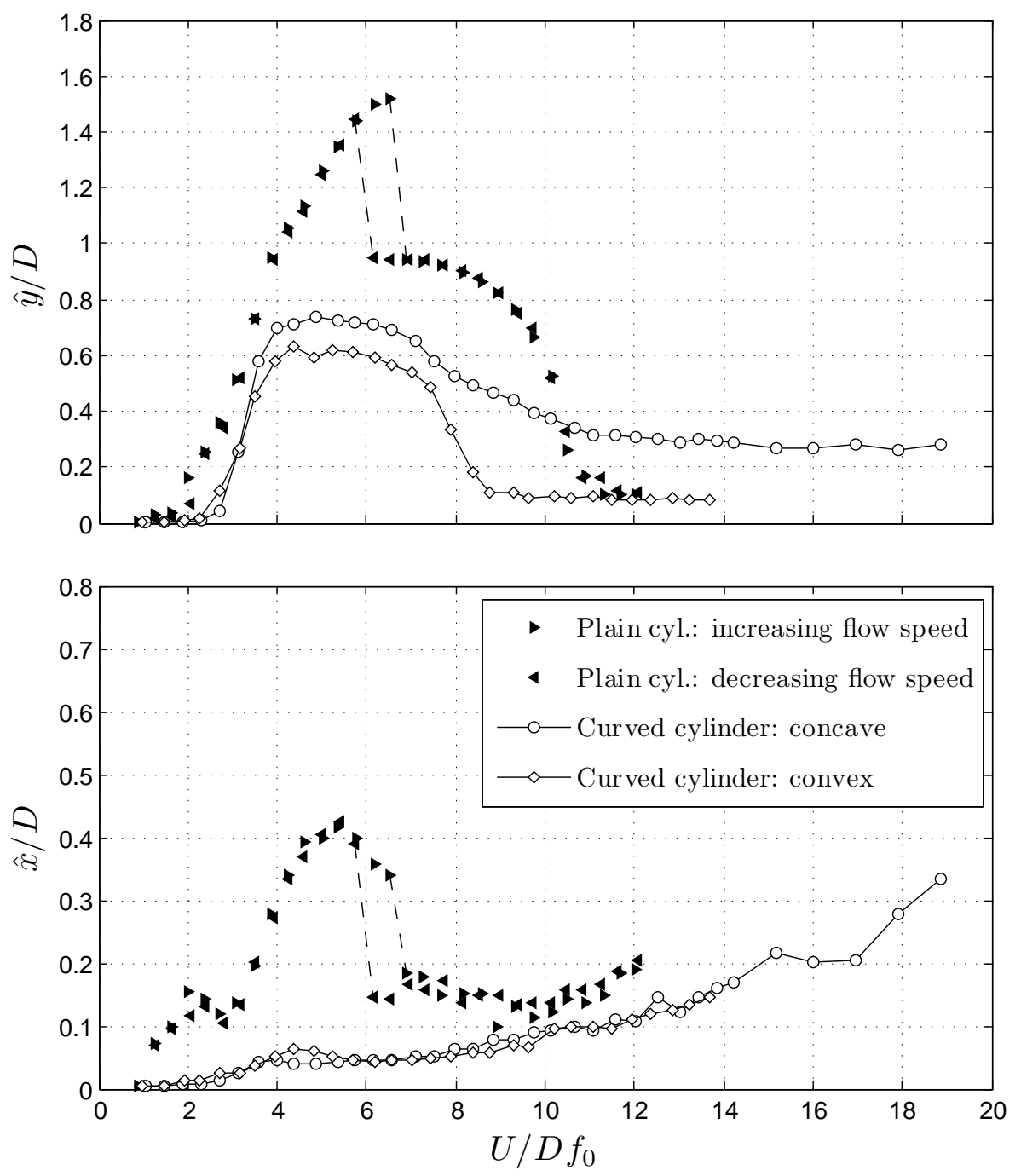

FIGURE 2. Cross-flow $(\hat{y} / D)$ and streamwise $(\hat{x} / D)$ amplitude of vibration versus reduced velocity for a straight cylinder and a curved cylinder in convex and concave configurations.

trajectories obtained for the three experiments. The straight cylinder presents distinct eight-shape figures typical of 2 dof VIV. On the other hand, trajectories for both configurations of the curved cylinder reveal that the streamwise displacement is greatly reduced when compared to the straight cylinder. Both concave and convex cases show very little movement in the streamwise direction for the whole range of reduced velocity. Another interesting observation relates the movement of both curved cylinders. It is clear that for reduced velocities higher than 10 the convex cylinder shows little displacement in both direction, while in the concave case vibrations are sustained until the end of the experiment.

The question to be answered is concerned with the fact that the convex configuration is able to drop down to a cross-flow amplitude of 0.1 while the concave configuration sustains vibration around 0.35 for high reduced velocities. We believe this distinct behaviour between the convex and the concave configurations can only be related to the wake interference happening in the lower half of the curvature due to perturbations generated in the horizontal section when it is positioned upstream.

PIV measurements of the flow field along the length of the cylinder are presented in Assi et al. [9] and support the discussion that follows. In the concave configuration the horizontal part of the cylinder is placed upstream of the curved and vertical parts. The approaching flow encounters a circular blunt leading edge with a clear separation region around the circumference. The flow that separates at the leading edge tends to create a separation 

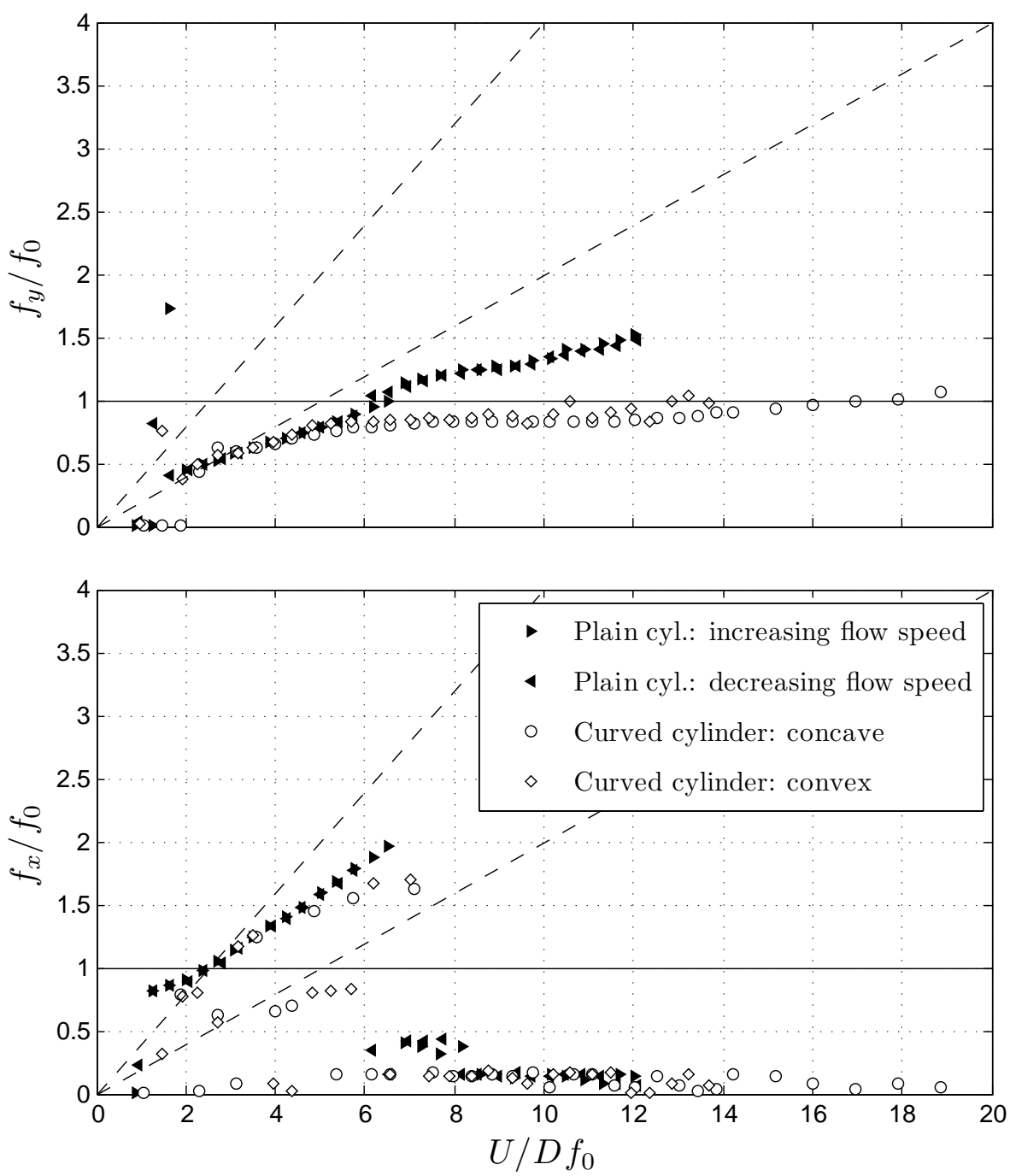

FIGURE 3. Cross-flow $\left(f_{y} / f_{0}\right)$ and streamwise $\left(f_{x} / f_{0}\right)$ dominant frequency of vibration versus reduced velocity for a straight cylinder and a curved cylinder in convex and concave configurations.

bubble and latter reattach along the horizontal section of the cylinder. Because the cylinder already presents cross-flow and streamwise vibrations, the three-dimensional separation bubble will not find a stable configuration nor a definite reattachment region, instead it will develop a periodic behaviour that may result in three-dimensional vortices being shed downstream, reaching the other parts of the cylinder.

The fluid-elastic mechanism behind the response is not yet clear, although the authors suggest it might be related to some kind of buffeting excitation due to the disturbed flow from the upstream horizontal part. We believe this interaction between the disturbed flow from the upstream horizontal part with the curved and vertical parts is responsible for sustaining a small level of vibration that resembles a buffeting-like mechanism. We suggest such an interaction may be happening in two forms:

(i) Vortices generated along the horizontal section may impinge on the curved part generating impulses in the same manner that large eddies of turbulence induce buffeting on elastic structures.

(ii) The disturbed flow from the horizontal part may be disturbing and disrupting the vortex shedding mechanism from the curved and vertical sections, for example uncorrelating the vortex shedding mechanism in a curved region of the cylinder near the horizontal part.

Of course both mechanisms suggested above may also be occurring simultaneously or it may not even be possible to explain them separately.

Evidence that a buffeting-like phenomenon might be 


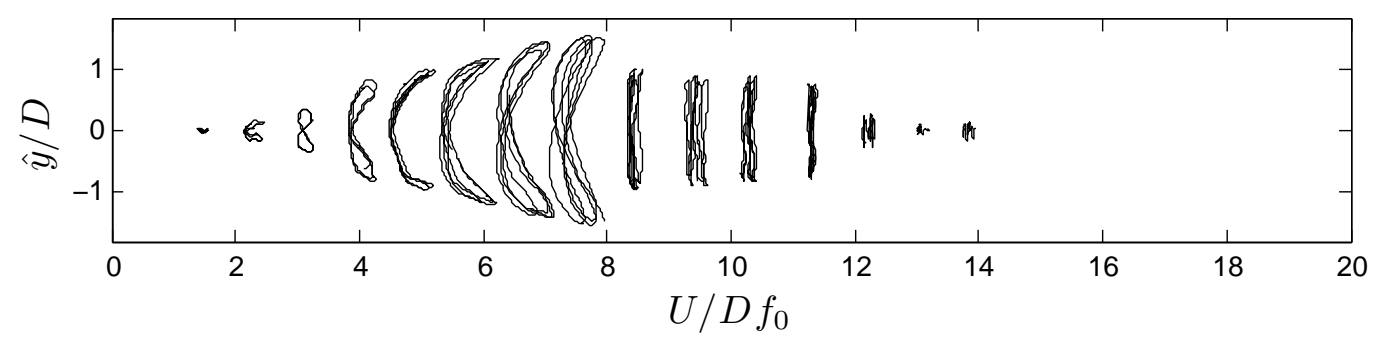

(a) Straight cylinder

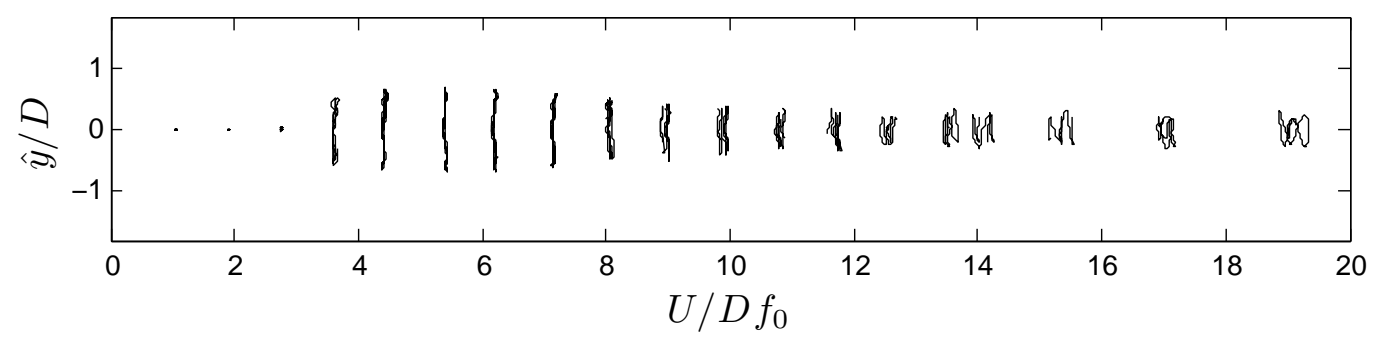

(b) Curved cylinder: concave configuration

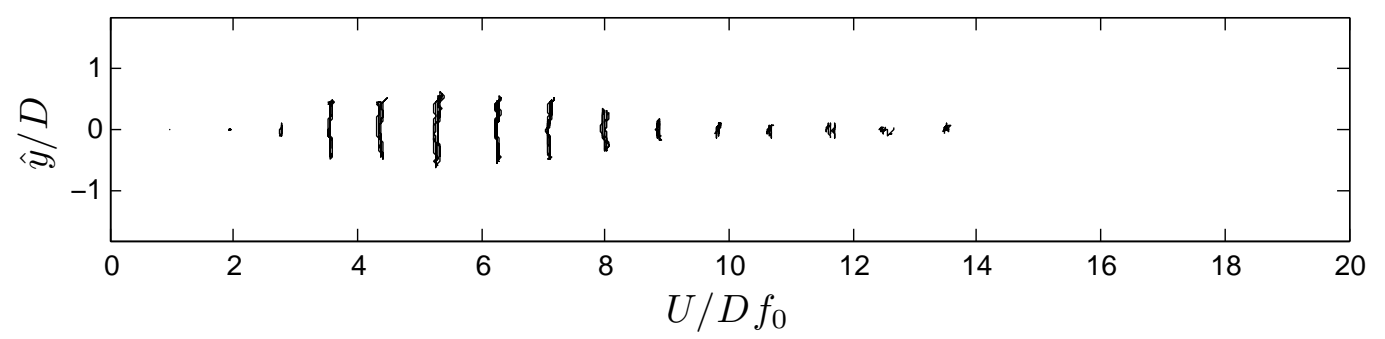

(c) Curved cylinder: convex configuration

FIGURE 4. Response trajectories of motion for a straight cylinder (a) and a curved cylinder in concave (b) and convex (c) configurations.

occurring is that the streamwise vibration shows a considerable increase in amplitude with increasing flow speed further out of the synchronisation range. Figure 4(b) also reveals that these vibrations are not harmonic and may even be chaotic, another evidence supporting the buffeting-excitation hypothesis.

Nevertheless, at this stage, we can only speculate about the flow-structure interaction that produced different responses for a concave and convex cylinder. This is being investigated by PIV measurements of the separated flow around the horizontal section and its interference with the vortex-shedding of the curved section.

\section{CONCLUSIONS}

We have investigated the VIV response of a curved cylinder in a concave and convex configurations regarding the approaching flow. We conclude that:

(i) In general terms, a curved cylinder presents a lower peak of amplitude of vibration in both the cross-flow and streamwise direction when compared to a straight cylinder. Nevertheless, a considerable level of in-line vibration not attributed to VIV was observed for reduced velocity as high as 18 .

(ii) Although the peak amplitude is reduced, a curved cylinder may present a significant level of vibration that is sustained for higher values of reduced velocity beyond the end of the typical synchronisation range.

(iii) The concave configuration shows a considerable level of cross-flow vibration around $\hat{y} / d=0.35$ up to the highest reduced velocity performed in this experiment.

(iv) We suggest that the flow-structure interaction mechanism that differentiates the concave form the convex response is a type of buffeting excitation with its origin in the disturbed flow that separates from the horizontal part located upstream.

A better understanding of the mechanism behind this phenomenon could be obtained by performing measurements of the flow along the horizontal part up to the curved section. This is being investigated employing PIV and should be published in the near future. 


\section{ACKNOWLEDGEMENTS}

The authors wish to acknowledge the support of FAPESP (São Paulo State Research Foundation) and "The Sir David Anderson Award" from the University of Strathclyde.

\section{REFERENCES}

[1] Miliou, A., De Vecchi, A., Sherwin, S.J., Graham, M.R. 2007 Wake dynamics of external flow past a curved circular cylinder with the free stream aligned with the plane of curvature J. Fluid Mech., 592, 89-115.

[2] Assi, G.R.S., Meneghini, J.R., Aranha, J.A.P., Bearman, P.W., Casaprima, E. 2006 Experimental investigation of flow-induced vibration interference between two circular cylinders. J. Fluids and Structures, 22, 819-827.

[3] Freire, C.M., Meneghini, J.R. 2010. Experimental investigation of VIV on a circular cylinder mounted on an articulated elastic base with two degrees-of-freedom. In the proceedings of BBVIV6 - IUTAM Symposium on Bluff Body Wakes and Vortex-Induced Vibrations, 2010, Capri, Italy.

[4] Assi, G.R.S., Bearman, P.W and Kitney, N 2009 Low Drag Solutions for Suppressing Vortex-Induced Vibration of Circular Cylinders. J. Fluids and Structures 25, 1-10.

[5] Assi, G.R.S., Bearman, P.W., Kitney, N., Tognarelli, M.A. 2010 Suppression of Wake-Induced Vibration of Tandem Cylinders with Free-to-Rotate Control Plates. J. Fluids and Structures, 26, 1045-1057.

[6] Freire, C.M., Korkischko, I., Meneghini, J.R. 2009 Development of an elastic base with tow degrees of freedom for VIV studies. In the proceedings of COBEM 2009 - 20th International Congress of Mechanical Engineering, 2009, Gramado, Brazil.

[7] Freire, C.M., Korkischko, I., Meneghini, J.R. 2011 Definning a parameter of effectiveness for the suppression of vortex-induced vibration. In the proceedings of OMAE2011 - 30th International Conference on Ocean, Offshore and Arctic Engineering, 2011, Rotterdam, The Netherlands.

[8] Assi, G.R.S., Bearman, P.W., Rodrigues, J.R.H., Tognarelli, M. 2011 The effect of rotational friction on the stability of short-tailed fairings suppressing vortex-induced vibrations. In the proceedings of OMAE2011, 30th International Conference on Ocean, Offshore and Arctic Engineering, Rotterdam, The Netherlands.

[9] Assi, G.R.S., Freire, C.M., Korkischko, I., Srinil, N. 2012 Experimental investigation of the flow-induced vibration of a curved circular cylinder. To appear in the proceedings of FIV2012 - 10th International Conference on Flow-Induced Vibration and Noise, 2012, Dublin, Ireland. 